PLANIFIER ET CONTROLER SES ACTIONS POUR ATTEINDRE UN OBJECTIF Présentation d'un dispositif pédagogique basé sur une pratique sportive au sein d'un master scientifique

Isabelle Pouliquen, Université Paul Cézanne, Marseille

Michel Calmet, Université de Montpellier

Résumé :

Après plusieurs années de fonctionnement dans un contexte de certification ISO 9001, une perspective d'amélioration continue, les spécificités du statut d'apprenti de nos étudiants et la volonté de professionnalisation de la formation ont conduit l'équipe pédagogique du Master Qualité à envisager son programme dans une approche systémique et à y insérer un module dit de développement personnel. Dans ce dernier; un dispositif pédagogique basé sur une pratique sportive, a été intégré. L'objet est de favoriser les prises de conscience et de développer un certain nombre de compétences que les apprenants doivent réutiliser dans leur approche et leur assimilation d'autres modules plus théoriques. Trente étudiants en master 1 "Analyse et Qualité" à la Faculté des Sciences et Techniques de I'Université Paul Cézanne Aix Marseille III, ont ainsi pratiqué une forme d'escrime japonaise pendant 10 heures réparties sur une semaine, aucun d'entre eux ne connaissait cette activité. Le comportement normal du débutant en combat apparaît comme "désordonné" et les premières actions réussies ressortent comme des faits rationnels brefs. Le but de ce dispositif était de s'appuyer sur l'analyse de ces faits rationnels notamment pour aborder la nécessité de planifier les actions pour atteindre un objectif (ici, toucher sans être touché). L'enquête de satisfaction menée à la fin de la formation et les travaux écrits rendus par les étudiants montrent que ce but est atteint. Les étudiants soulignent de plus l'intérêt des échanges et de la coopération au cours des exercices mais aussi l'utilisabilité des apprentissages réalisés dans le contexte global de leur formation. Une de nos perspectives est de continuer les enquêtes pour connaître quand et comment ces compétences acquises sont de surcroit transférées dans la vie professionnelle. 


\section{Introduction, mise en contexte}

L'objectif général du Master mention Qualité est de former des professionnels aptes à concevoir, mettre en place et animer une démarche système de management Qualité, hygiène, sécurité, environnement, (QHSE) ou intégré, ou encore plus actuelle d'optimisation des performances (grands groupes industriels).

Aujourd'hui la mise en place et la maîtrise de systèmes de management sont intégrés au fonctionnement des organisations et nécessitent des acteurs en mesure d'avoir une vision globale de ces organisations, d'apporter leurs connaissances au niveau stratégique, de mettre en place les dispositifs de mesure des performances et d'en présenter les résultats, tout en animant la démarche au niveau opérationnel. Planification, communication, organisation, analyse et modélisation de processus, sont des pierres angulaires de cette formation et des éléments constitutifs de la roue de Deming.

L'équipe pédagogique, majoritairement constituée de professionnels du domaine QHSE, fait régulièrement évoluer le programme de la formation depuis sa création (1986). Ce programme est depuis quelques années basé sur l'objectif pour nos étudiants d'acquérir les compétences liées aux concepts suivants :

- Appréhender, comprendre le fonctionnement interne d'une organisation

- Avoir un comportement adapté-: favoriser le dialogue, savoir être, manager

- Connaître et savoir appliquer-: les principes qualité, hygiène, sécurité et environnement, les outils qualité, les référentiels, la gestion de projet, des outils techniques ...

- Situer l'organisation par rapport : aux pouvoirs publics, au marché

Les résultats notamment en termes de placement des étudiants sont conformes aux objectifs du système de management de la qualité du Master. Cependant, le fait que les étudiants sont d'une part de formation initiale scientifique donc peu voire pas formés aux sciences humaines, d'autre part en contrats d'apprentissage et donc en alternance ( 1 mois / 1 mois durant 12 mois) entre l'université et l'entreprise nous ont conduit à souhaiter accélérer le passage des concepts théoriques à la pratique, et à permettre aux étudiants de concrétiser, de pouvoir appliquer et de rendre rapidement opérationnels les éléments conceptuels de la formation.

En effet, malgré la mise en place de projets, de travaux de groupe, d'études de cas....la progression constatée, surtout lors des premières périodes de l'année, à la fois dans la capacité des étudiants à mettre en pratique les apprentissages mais aussi à adopter une attitude de participation active et réactive durant les enseignements, reste un frein à leur capacité à profiter pleinement des premières périodes de formation en entreprise, mais aussi à tirer le meilleur parti du potentiel et de l'expérience des intervenants.

L'apport théorique décrit sur le schémal suivant sur les progressions dans les apprentissages (Cleeremans et Destrebecqz, 2002) nous a conduit à intégrer un module dit de développement personnel comme élément pivot de la formation. Ce module comporte plusieurs éléments visant à atteindre plus rapidement la phase d'automaticité. 


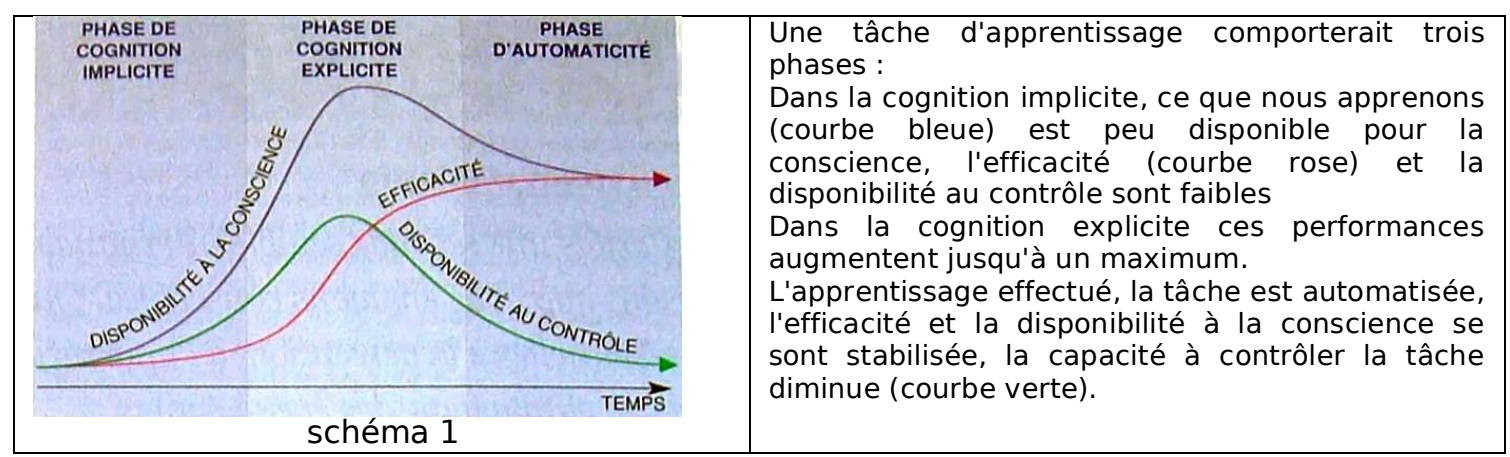

Dans ce module, de nouveaux dispositifs pédagogiques sont mis en œuvre, et notamment la formation par une pratique sportive.

L'utilisation de pratiques sportives pour développer la cohésion et la compréhension dans un groupe est largement connue, utilisée et dans certains cas décriée, dans le monde professionnel comme dans certaines écoles. Notre objectif ici est, au-delà des résultats sur le groupe, de permettre le transfert de compétences acquises par la pratique sportive vers des compétences en management de la qualité et en management de projet. II s'agit de travailler non seulement sur l'effet de groupe mais en groupe sur le comportement individuel des membres du groupe pour faciliter la traduction de concepts en activités et pour accentuer la prise de conscience et l'assimilation de concepts. Ce résultat n'a, à notre connaissance pas été décrit dans la littérature, mais nous a semblé constituer un objectif réalisable.

Considérations générales sur la pratique sportive

Que peuvent apporter des pratiques sportives dans un contexte "qualité", pour atteindre un objectif et pour communiquer?

Dans les sports en général on peut considérer que l'empathie et l'altérité sont toujours présentes, mais il faut remarquer que:

La compétition est reliée à la concurrence et au classement,

L'entrainement est relié à la coopération et l'échange (ce qui ne signifie pas supprimer la confrontation).

La réalisation d'une action motrice (individuelle ou collective) n'est pas le fait du hasard. Quelles que soient les approches (machine organisée/machine organisante [Paillard, 1978]; traitement de l'information; écologique; cognition située [Hauw, 2002]) et quelles que soient les activités physiques sportives et artistiques, l'individu "entier" (système complexe ou cognition et motricité sont articulés) est engagé dans la réalisation d'une action dans un contexte. Les différents segments (membres supérieurs, tronc, bassin, membres inférieurs) doivent être mobilisés par rapport à une action "représentative type" mémorisée (gymnastique), et par rapport à une opposition (sports collectifs; sports de combat) pour marquer des points (figures et difficultés en gymnastique) ou marquer un point (but en sports collectifs; avantage ou point décisif en sports de combat). Certains sports, nautiques par exemple, demandent de coordonner une machine (le bateau) un équipage et des conditions extérieures (vents, houles, courants, bateaux adverses).

II s'agit de planifier des actions pour atteindre un objectif, les pratiques sportives répondant en cela à la roue de Deming (Plan, Do, Check, Act...).

Notre présentons ici les retombées de 12 heures de formation (10 heures de pratique sportive et 2 heures de théorie). 


\section{Méthodologie et recueil de données}

Description de l'activité sportive

Pour des raisons de mise en œuvre (proximité des installations dans l'université, homogénéité du niveau de non connaissance des activités de combat de type escrime par les étudiants, matériel disponible) l'activité sportive retenue est une forme d'escrime japonaise adaptée pour être enseignée à l'école: le kendoscolaire. C'est une pratique avec un bâton rigide recouvert de mousse. La protection (armure, casque et gants) a été placée sur le bâton. Celui-ci est donc recouvert d'une mousse épaisse et a été raccourci. Son poids est d'environ $400 \mathrm{~g}$. La gestuelle est sécurisée (saisie à deux mains du bâton, touche posée à l'impact), on peut toucher tout le corps adverse (avec le côté du bâton, pas avec l'extrémité) sauf la tête, la poitrine et l'entrejambe. Cela permet de mettre en avant la prise d'information visuelle (le judo demande une information kinesthésique, plus longue à rendre opérationnelle). Ces armes sécurisées rendent la pratique attractive (symbolique et affordance). Le risque de blessures amenant des gênes professionnelles est réduit. II s'agit de toucher l'autre (touche posée à l'impact) sans être touché. La mise en action du bâton repose sur la vitesse et la précision et non sur la force et la puissance. La distance (médiée par l'arme) entre les combattants, est plus grande qu'en lutte ou qu'en boxe.

L'intérêt des sports de combat réside dans le fait que le combattant peut être assimilé à un système entier et complexe dans lequel il va falloir:

Décider de l'objectif

Décider des processus à mobiliser et de leurs mises en œuvre

Contrôler leurs déroulements

Poursuivre / Remédier

La connaissance de la réussite ou non de l'action est immédiate, elle implique la personne dans les 4 étapes ci-dessus.

Les actions peuvent être répétées, on peut tester facilement différentes actions.

L'action de formation repose sur quatre points:

1. D'un combat global, on passe à une action ordonnée par le combat "à mémoire". Le combat devient "à mémoire" Iorsqu'une touche est réussie deux fois de façon identique. L'opposition est alors interrompue.

2. Les deux combattants doivent refaire en coopération ce qui a été réussi, en recréant le contexte du combat. L'action, considérée comme un processus, est donc analysée et verbalisée.

3. L'objet du combat devient alors d'être capable de montrer aux autres ce que l'on a réussi spontanément dans le combat, donc de transmettre cette acquisition.

4. Les solutions trouvées (touches réussies) sont décrites sous forme de règles d'actions. La comparaison des différentes solutions et des règles d'actions permet une généralisation en principes d'actions. Cette partie est traitée grâce à un apport théorique sur la continuité des actions (enchainements, feintes, touches) en référence à l'intelligence artificielle et aux chainages des règles de production dans un système (base de connaissances, base de faits, moteur d'inférences). Le schéma 2 illustre ce type de chaînage : 


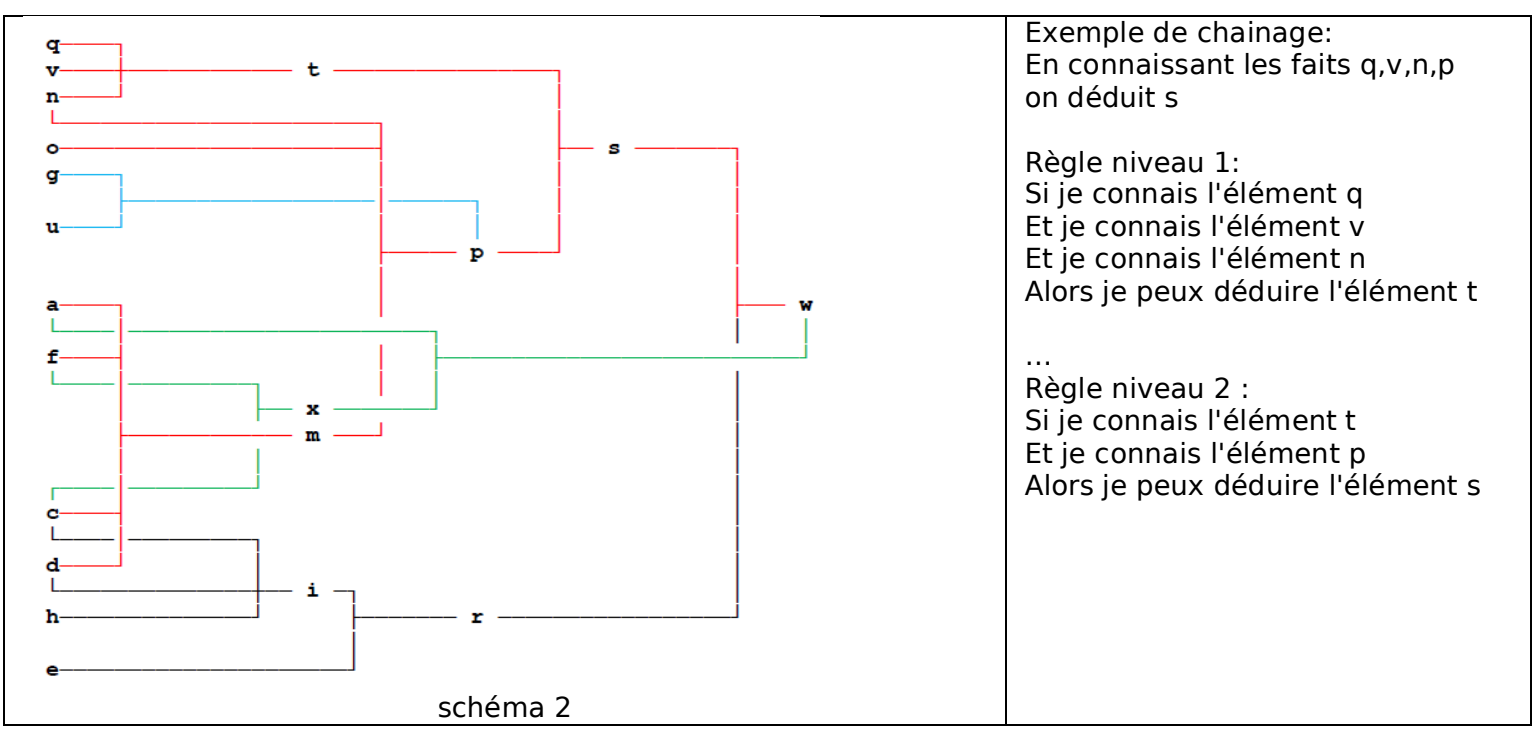

Les exercices pratiqués permettent de découvrir l'activité à partir de situations de travail, mais aussi de stabiliser les apprentissages et d'organiser des combats d'entraînement ou d'évaluation. Les solutions que peuvent trouver les combattants sont multiples. Ils sont donc amenés dans la confrontation à élaborer une intervention, à retrouver ou réinventer des solutions. Répété, le geste réussi spontanément est mieux compris et retenu, c'est un élément de rationalité (action courte réussie) dans un ensemble peu connu et confus (combat long). La confrontation à l'autre pendant l'entrainement permet des échanges, le changement régulier de partenaire d'entrainement permet de mieux connaître et comprendre les autres.

Après quelques heures de pratique, les contraintes pédagogiques fixées lors des combats (toucher une seule zone du corps, ou indiquer au partenaire quelle zone il doit toucher) permettent de comprendre que toucher est une action volontaire qui n'est pas due au hasard. II devient nécessaire de planifier ses actions pour atteindre la cible.

L'objectif de la formation est de faire comprendre cette nécessité pour que les étudiants puissent la réinvestir dans leur cursus universitaire et dans le monde professionnel.

En complément de la pratique sportive, deux heures sont consacrées aux théories de l'apprentissage et à la continuité/enchaînement des actions comme dans un système à base de règles en intelligence artificielle.

Les étudiants rendent un travail écrit concernant l'ensemble de la formation et répondent à une enquête de satisfaction.

Enquête de satisfaction.

4 questions générales (âge, genre, ordinateur, accès à l'internet)

9 questions, sur le cours et son enseignement (4 niveaux d'appréciation)

2 questions ouvertes (ce qui vous a plu, ce qui vous a déplu)

Le questionnaire est rempli en moins de $7 \mathrm{~min}$, les 13 premières questions font l'objet d'une analyse descriptive (moyenne, écart type, pourcentages)

Les contenus des deux dernières questions sont classés en thèmes.

Discussions, perspectives

Quelques travaux d'étudiants seront analysés et présentés sous forme d'étude de cas pour engager des perspectives. 


\section{Résultats}

L'enquête

\begin{tabular}{|c|c|c|c|c|c|}
\hline Date des réponses & Du $10 / 3$ au $20 / 3$ & & & & \\
\hline Genre & $21 \mathrm{~F}$ & $12 \mathrm{M}$ & 35 rép. sur 36 soit : $97 \%$ & & \\
\hline Age moyen & $22,7 \pm 2,7$ & $24,2 \pm 5,4$ & & & \\
\hline Possédez-vous un ordinateur personnel? & $94,3 \%$ Oui & $0 \%$ Non & & & \\
\hline Avez-vous internet ADSL à votre domicile? & $85,7 \%$ Oui & $8,6 \%$ Non & & & \\
\hline Faites-vous du sport en dehors de l'Université ? & $8,6 \%$ Jamais & $48,6 \%$ Parfois & $20 \%$ Souvent & $17,1 \%$ Très souvent & \\
\hline $\begin{array}{l}\text { Cet enseignement vous semble-t-il avoir sa place } \\
\text { dans les maquettes de votre diplôme? }\end{array}$ & $28,6 \%$ Oui & $57,1 \%$ Plutôt oui & 2,9\% Plutôt non & $0 \%$ Non & $5,7 \%$ Je ne sais pas \\
\hline $\begin{array}{l}\text { La forme de cet enseignement vous semble-t-elle } \\
\text { pertinente? }\end{array}$ & $45,7 \%$ Oui & $40 \%$ Plutôt oui & $8,6 \%$ Plutôt non & $0 \%$ Non & $0 \%$ Je ne sais pas \\
\hline $\begin{array}{l}\text { Globalement l'enseignement vous paraît-il en } \\
\text { adéquation avec le cursus que vous avez choisi ? }\end{array}$ & $25,7 \%$ Oui & $60 \%$ Plutôt oui & $8,6 \%$ Plutôt non & $0 \%$ Non & \\
\hline $\begin{array}{l}\text { Le contenu des cours vous paraît-il adapté à votre } \\
\text { niveau de connaissance? }\end{array}$ & $62,9 \%$ Oui & 28,6\% Plutôt oui & 2,9\% Plutôt non & $0 \%$ Non & \\
\hline $\begin{array}{l}\begin{array}{l}\text { Etes-vous satisfait du déroulement des cours } \\
\text { (progressivité et interactivité) ? }\end{array} \\
\end{array}$ & $65,7 \%$ Oui & $25,7 \%$ Plutôt oui & 2,9\% Plutôt non & $0 \%$ Non & \\
\hline $\begin{array}{l}\text { Etes-vous satisfait des documents (supports de } \\
\text { cours) mis à votre disposition sur internet? }\end{array}$ & $34,3 \%$ Oui & $54,3 \%$ Plutôt oui & 2,9\% Plutôt non & $2,9 \%$ Non & \\
\hline Quelle note pensez-vous obtenir au travail écrit? & $13,8 \pm 1,8$ & $17 \mathrm{Max}$ & $11 \mathrm{Min}$ & & \\
\hline Avez-vous trouvé l'enseignant réactif? & $85,7 \%$ Complètement & $5,7 \%$ Partiellement & $0 \%$ Pas vraiment & $0 \%$ Pas du tout & $2,9 \%$ Sans avis \\
\hline Ce qui vous a plu dans le cours & \multicolumn{5}{|c|}{$\begin{array}{l}\text { Mieux connaitre les autres; L'idée de réfléchir à ce qu'on va faire avant de l'appliquer; Découverte des techniques/tactiques par } \\
\text { nous même }\end{array}$} \\
\hline Ce qui vous a déplu dans le cours & \multicolumn{5}{|c|}{ Cours trop rapprochés (étaler l'enseignement sur l'année); } \\
\hline
\end{tabular}

Les différents taux de satisfaction se retrouvent dans les propos écrits des étudiants. Voici deux exemples concernant la planification $(P)$ et la présentation de règles d'action (RA):

\section{Caroline :}

(P)

"C'est plus précisément lorsque nous devions toucher une seule zone pour marquer des points que nous avons commencé à planifier des actions et à mettre en pratique les règles de combats. II est vrai que lorsque l'objectif est fixé, il est plus facile de planifier des actions pour l'atteindre, ce qui conduit plus facilement à la réussite. A partir de là, nous avons assimilé l'importance de la planification en observant que chacune de nos actions pouvaient entrainer plusieurs sortes de réactions chez l'adversaire et que nous pouvions les envisager pour mieux atteindre notre objectif.

En mettant en avant le lien entre la planification des actions et l'atteinte des objectifs dans le sport, il est également possible de transposer ce concept dans le cadre de la formation. En effet, la réussite d'un projet réside dans l'organisation de celui-ci et dans la planification des actions et des tâches à effectuer. La planification d'un projet permet tout d'abord d'estimer le temps que chaque tâche peut nécessiter et permet d'avancer par étape...

A une échelle moindre dans le temps, le sport nous montre que le terme de planification est assez large. II n'induit pas forcément l'utilisation d'outils spécifiques mais incite à prévoir des actions quelles qu'elles soient dans le but de satisfaire à un objectif précis."

(RA)

"1) Si les armes sont en contact et que l'adversaire exerce une force rigide, Alors je peux faire glisser mon arme en arrière pour la dégager et faire une touche sur le côté opposé de l'adversaire 
2) Si les armes sont en contact et que l'adversaire exerce une force souple, Alors je peux faire tourner l'arme de mon adversaire avec mon arme et faire une attaque sur une partie libre.

3) Si je distrais l'adversaire avec une attaque haute ou basse (feinte), et que celui-ci contre mon attaque à l'endroit que je souhaitais, alors je peux attaquer sur la partie libre opposée.

4) Si l'adversaire tente une attaque "barbare" alors je peux esquiver et le toucher à l'épaule."

Alix:

(P)

"Si nous comparons la planification d'actions au cours d'une pratique sportive et dans une entreprise, il s'agit dans les deux cas de mobiliser des forces (individuelles ou collectives) en vue d'atteindre un objectif. II faut être vif, réactif, faire preuve d'organisation, de réflexion avant l'action et d'anticipation...

Des efforts (physique et mental) sont demandés lors de la pratique de ce sport car il faut planifier nos mouvements et riposter (ou contrer les attaques)...

...Cette même personne a ensuite dû montrer à l'ensemble du groupe comment elle est arrivée à toucher deux fois son partenaire au même endroit, puis chacun a dû refaire l'enchaînement réalisé par cette personne, avec son partenaire. II y a donc eu partage des informations...

Nous pouvons constater que l'apprentissage et la coopération sont étroitement liés.

En entreprise, la stratégie peut s'établir en groupe, chacun apportant ses idées, ses commentaires quant au déroulement des étapes qui permettront la réalisation d'un projet. Cette coopération permet également d'avoir un avis plus général sur la question abordée et ainsi d'avancer plus efficacement. Cependant le respect envers autrui est très important, chaque personne a le droit de s'exprimer."

(RA)

Exemple 1

Règle 1 :

Si je viens battre (taper) fort l'arme de mon adversaire,

Et si je l'écarte d'un côté,

Alors l'autre côté est libre.

Règle 2 :

Si l'autre côté est libre,

Alors je peux le toucher de ce côté.

Exemple 2

Règle 1 :

Si mon adversaire frappe fort pour repousser mon bâton,

Et si j'esquive son attaque en enlevant mon bâton,

Alors mon adversaire est emporté par la force de frappe.

Règle 2 :

Si mon adversaire est emporté par l'élan du côté gauche,

Alors son côté droit est libre.

Règle 3 :

Si le côté droit de mon adversaire est libre,

Alors je peux le toucher à l'épaule droite. 


\section{Discussion, Perspectives}

Intérêt de ce type de formation:

L'analyse des retours des étudiants montrent bien qu'ils ont saisi les bases de l'organisation par projet. Au-delà des outils (Pert, Gantt) qu'ils ont au préalable eu à utiliser dans des travaux de groupe, l'importance de la planification des actions et des tâches, du partage des informations, de la coopération ont gagné du sens. La traduction de l'action réussie de façon verbale puis en chainage logique d'actions est une expérience qu'ils pourront mettre à profit dans l'analyse et la description des processus en entreprise, qui leur facilitera la compréhension de ces mêmes notions de processus, clefs en management qualité.

Le fait de montrer au groupe l'action réussie est un entraînement à la transmission de connaissances, activité également importante dans ce domaine, et inversement chaque personne du groupe est amenée à observer l'action reproduite et à l'analyser. Le management de la qualité comprend en parallèle ce type de taches d'observation et d'analyse, notamment lors de phases de reengineering de processus, et les exercices réalisés peuvent permettre aux étudiants d'avoir une approche réflexive plutôt qu'approche intuitive.

De récentes études dans le monde industriel ont montré que la confiance, l'empathie et la capacité d'un "système humain" à pallier un dysfonctionnement/déficience en utilisant d'autres procédures pour atteindre l'objectif sont des gages de productivité (Pentland, 2011).

Perspectives:

Poursuivre le travail par une analyse détaillée des textes des étudiants

Etudier quand et comment ces compétences acquises sont transférées dans la vie professionnelle.

\section{Références}

2002, Cleeremans A, Destrebecqz A., Apprentissage et conscience, Pour la Science 302, 105-109

1978, Paillard J., EPS interroge un psychophysiologiste, Revue EP.S 154 et 155 Paris.

2002, Hauw D., Enseignement et apprentissage : une vision située. Revue EP.S, 298, 54-58

2011, Pentland A, Les signaux non verbaux de la communication, Pour la science 399, 44-50. 